\title{
Enhancement of pure volume negative ion production using a grid bias method or a magnetic filter method ${ }^{\text {a) }}$
}

\author{
Yasuhiro Jyobira, Daisuke Ito, and Osamu Fukumasa ${ }^{\text {b) }}$ \\ Graduate School of Science and Engineering, Yamaguchi University, Tokiwadai 2-16-1, Ube 755-8611, Japan
}

(Presented 27 August 2007; received 27 August 2007; accepted 8 October 2007; published online 30 January 2008)

Volume production of hydrogen negative ion $\mathrm{H}^{-}$is studied in pure hydrogen plasmas using a grid bias method for plasma parameter control. The purposes of the present study are as follows. One is to investigate the possibility of controlling plasma parameters with a grid bias method in dc discharge plasmas; the other is to realize efficient negative ion production in $\mathrm{H}_{2}$ plasmas and to discuss the difference in plasma parameters control and $\mathrm{H}^{-}$production between the grid bias method and the usual magnetic filter method. The relationship between plasma parameters and extracted $\mathrm{H}^{-}$ ion currents is discussed. It is confirmed that both high and low electron temperature $T_{e}$ plasmas are produced in the separated regions when the grid is negatively biased. The negative ion production depends strongly on the grid potential and related plasma conditions. Within certain plasma conditions, $\mathrm{H}^{-}$production with grid bias method is much higher than one with magnetic filter method. (C) 2008 American Institute of Physics. [DOI: 10.1063/1.2805368]

\section{INTRODUCTION}

In the design of a neutral beam injection (NBI) system for future large fusion devices such as the ITER, sources of $\mathrm{H}^{-}$or $\mathrm{D}^{-}$negative ions are required for efficient generation of neutral beams with energies above $\approx 100 \mathrm{keV} /$ nucleon. In volume $\mathrm{H}^{-}$source, most of the $\mathrm{H}^{-}$ions are generated by the dissociative attachment of slow plasma electrons $e_{s}$ (electron temperature $T_{e} \sim 1 \mathrm{eV}$ ) to highly vibrationally excited hydrogen molecules $\mathrm{H}_{2}\left(v^{\prime \prime}\right)$ (effective vibrational level $v^{\prime \prime}$ $\geqq \geqq 5-6$ ). These $\mathrm{H}_{2}\left(v^{\prime \prime}\right)$ are mainly produced by collisional excitation of fast electrons with energies in excess of 15-20 eV. Namely, $\mathrm{H}^{-}$ions produced by the following twostep process: ${ }^{1}$

$$
\begin{aligned}
& \mathrm{H}_{2}\left(X^{1} \Sigma_{g}, v^{\prime \prime}=0\right)+e_{f}(E \sim 15-20 \mathrm{eV}) \\
& \rightarrow \mathrm{H}_{2}^{*}\left(B^{1} \Sigma_{u}, C^{1} \Pi_{u}, v^{\prime}\right)+e_{f}^{\prime}, \\
& \mathrm{H}_{2}^{*}\left(v^{\prime}\right) \rightarrow \mathrm{H}_{2}\left(X^{1} \Sigma_{g}, v^{\prime \prime}\right)+h \nu, \\
& \mathrm{H}_{2}\left(v^{\prime \prime} \geqq 5\right)+e_{s}\left(T_{e} \sim 1 \mathrm{eV}\right) \rightarrow \mathrm{H}^{-}+\mathrm{H} .
\end{aligned}
$$

Therefore, spatial control of electron energy distribution (i.e., $T_{e}$ ) is necessary. ${ }^{2,3}$ So far, the magnetically filtered multicusp ion source has been shown to be a promising source of high-quality multiampere $\mathrm{H}^{-}$ions. To enhance $\mathrm{H}^{-}$ion production and extracted $\mathrm{H}^{-}$ion currents, a small amount of cesium vapor is usually seeded into the above volume ion source. Therefore, ion source operation is rather complicated-to keep optimum surface condition of the plasma grid for surface production of $\mathrm{H}^{-}$ions.

\footnotetext{
Contributed paper, published as part of the Proceedings of the 12th International Conference on Ion Sources, Jeju, Korea, August 2007.

${ }^{b)}$ Author to whom correspondence should be addressed. FAX: +81-836-859401. Electronic mail: fukumasa@plasma.eee.yamaguchi-u.ac.jp.
}

Long lifetime ion sources are also required for future NBI systems. Microwave-discharge ion sources ${ }^{4}$ and rfdriven ion sources ${ }^{5,6}$ are promising as long lifetime ion sources because they have no filaments. Unfortunately, in those high-frequency plasmas, control of plasma parameters using the magnetic filter method is not as effective as in dc plasmas. On negative ion sources, therefore, another important and interesting aspect is to develop the ion sources without filaments, magnetic filter, and cesium injection.

The aims of the present study are as follows. The first is to study the application feasibility of a grid bias method ${ }^{7,8}$ for controlling plasma parameters, in particular $T_{e}$, in dc discharge $\mathrm{H}_{2}$ plasmas; the second is to discuss the difference in $T_{e}$ control and $\mathrm{H}^{-}$production between the grid bias method and the usual magnetic filter method. At first, using both the mesh grid bias method and the usual magnetic filter method, plasma parameter control in de discharge plasmas is studied. Preliminary results on the grid bias method are reported here. ${ }^{9}$ The relationship between the extracted $\mathrm{H}^{-}$ion currents and plasma conditions is also briefly discussed.

\section{EXPERIMENTAL SETUP AND PROCEDURE}

Figure 1 shows a schematic diagram of the newly designed experimental apparatus for studying the negative ion source with grid bias method. ${ }^{9}$ The source chamber $(20 \mathrm{~cm}$ in diameter) made of stainless steel is divided by a mesh grid (MG) into two parts, i.e., a source region and an extraction region. To produce both high and low electron temperature plasmas separately in the chamber, this MG which is $19 \mathrm{~cm}$ in diameter is placed. In the present experiment, three different mesh grids are used, i.e., No. 1 (16 mesh/in.), No. 2 (30 mesh/in.), and No. 3 (50 mesh/in.). Details are shown in Table I. Compared with the usual magnetically filtered multicusp ion source, in Fig. 1, the magnetic filter (MF) 


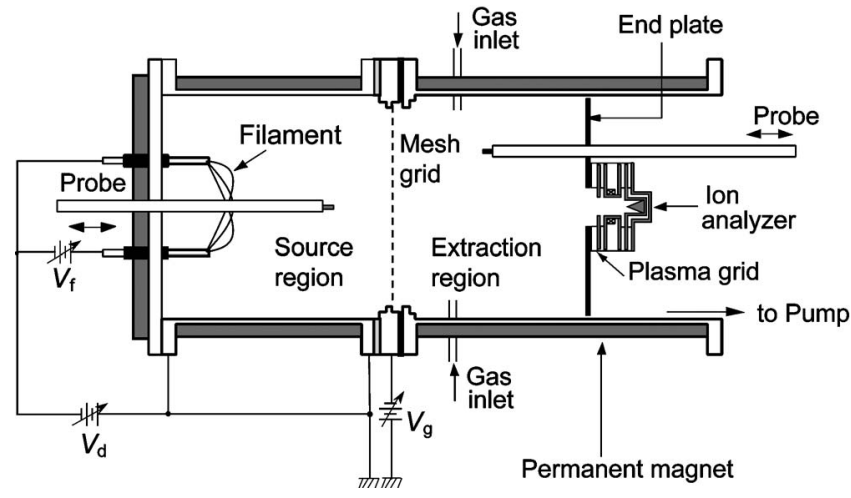

FIG. 1. Schematic diagram of the experimental apparatus.

flange is set instead of the MG flange. The MF consists of four rods. The diameter of each rod is $10 \mathrm{~mm}$ and the distance between two rods is $54 \mathrm{~mm}$. In the present experiment, the magnetic field intensity of the MF $\left(B_{\mathrm{MF}}\right)$ is set at 60 and $100 \mathrm{G}$. In the source region, steady-state $\mathrm{H}_{2}$ plasmas are produced by dc arc discharge between the chamber anode and the tungsten filament cathode.

Plasma parameters (electron density $n_{e}$, electron temperature $T_{e}$, plasma space potential $V_{s}$, and floating potential $\left.V_{f}\right)$ are measured by two Langmuir probes. They are axially moved from the MG. The negative ion currents $\left(I_{\mathrm{H}^{-}}\right)$are directly detected by a magnetic deflectiontype ion analyzer. The plasma grid has a single hole $(10 \mathrm{~mm}$ in diameter) through which negative ions were extracted from the ion source.

\section{EXPERIMENTAL RESULTS AND DISCUSSION}

\section{A. Plasma production and control}

First, we test the grid bias method for $\mathrm{H}^{-}$production in dc discharge $\mathrm{H}_{2}$ plasmas. ${ }^{9,10}$ Figure 2 shows a typical example of grid bias effect on plasma parameters in the extraction region for three different MGs. For all mesh MGs, there appears a decrease in $T_{e}$ with a decrease in grid biasing voltage $V_{g}$. In the extraction region, generation mechanism of low electron temperature plasma is as follows. In the extraction region, the neutral particles are ionized by higher energy electrons flowing from the source region through the grid. ${ }^{7,8}$ The electrons produced in the extraction region cannot be accelerated by the external electric fields because no additional heating power is fed into this region. Therefore, low energy electrons are generated in the extraction region.

The grid bias method is the method to control electrons transported electrostatically and is affected by the mesh size. ${ }^{7}$ By using three different MGs, the effect of mesh size is

TABLE I. Dimensions of the three meshes used in the present experiment.

\begin{tabular}{lcccc}
\hline \hline Mesh & $\begin{array}{c}\text { Mesh size } \\
(\text { mesh/in.) }\end{array}$ & $\begin{array}{c}\text { Diameter } \\
\text { of wire } \\
(\mathrm{mm})\end{array}$ & $\begin{array}{c}\text { Distance between } \\
\text { two wires } \\
(\mathrm{mm})\end{array}$ & $\begin{array}{c}\text { Geometric } \\
\text { transmittance }(\%)\end{array}$ \\
\hline No. 1 & 16 & 0.6 & 1 & 40 \\
No. 2 & 30 & 0.25 & 0.6 & 50 \\
No. 3 & 50 & 0.05 & 0.46 & 81 \\
\hline \hline
\end{tabular}
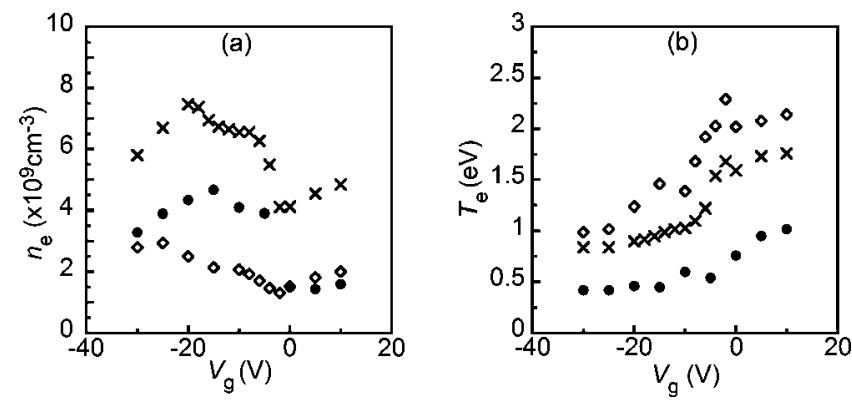

FIG. 2. (a) Electron density $n_{e}$ and (b) electron temperature $T_{e}$ for various grid potentials $V_{g}$ for the different mesh sizes of No. $1(\diamond)$, No. $2(\bullet)$, and No. $3(\times)$ at $z=-3.5 \mathrm{~cm}$ in the extraction region, where the end plate is set at $z_{\text {end }}=-8 \mathrm{~cm}$. Experimental conditions: discharge voltage $V_{d}=50 \mathrm{~V}$, discharge current $I_{d}=2 \mathrm{~A}$, and hydrogen gas pressure $p\left(\mathrm{H}_{2}\right)=0.4 \mathrm{~Pa}$.

also tested. We estimate the Debye length $\left(\lambda_{D}\right)$ from the typical plasma parameters (i.e., $n_{e} \simeq 1 \times 10^{10} \mathrm{~cm}^{-3}$ and $T_{e}$ $\simeq 2.0 \mathrm{eV}$ ) at $z=3.0 \mathrm{~cm}$ in the source region and obtain that $\lambda_{D} \simeq 0.1 \mathrm{~mm}$. When we suppose that sheath length is about several times the $\lambda_{D}$, distances between two wires of all meshes are within the sheath length. In the extraction region, $n_{e}$ with No. 3 mesh is higher than other two meshes (i.e., No. 1 and No. 2). This is caused mainly by the difference of transmittance. It is easy for electrons to cross the MG and enter into the extraction region as No. 3 mesh has high transmittance.

Figure 3 shows axial distributions of plasma parameters (i.e., $n_{e}$ and $T_{e}$ ) for two different controlling methods. The one is the mesh bias method and the other is the magnetic filter method. High energy electrons pass the MG (set at $z$ $=0 \mathrm{~cm})$ and enter into the extraction region. As a whole, $n_{e}$ increases in its value with $z$ and reaches the maximum value and then decreases while $T_{e}$ decreases in its value. With changing $V_{g}$, values of $n_{e}$ and $T_{e}$ are varied. Although this feature is not shown clearly in Fig. 3, that characteristic feature is well observed when the end plate is set far from the MG. By changing $V_{g}$ negatively, values of $n_{e}$ increase while $T_{e}$ decreases in its value. At any rate, with varying $V_{g}$ and the distance between the end plate (i.e., its position $z_{\text {end }}$ ) and the MG, plasma parameters can be controlled.

We have confirmed numerically that extraction probability of negative ions depends strongly on upstream distance from the extraction grid, ${ }^{11}$ i.e., the plasma end plate in the
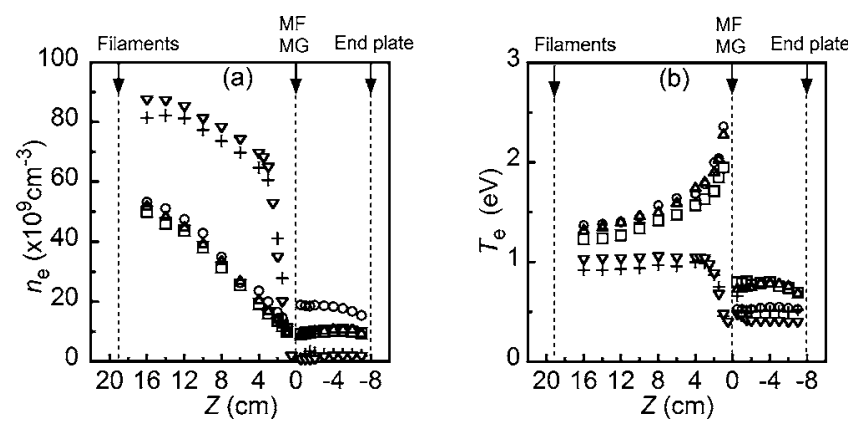

FIG. 3. Axial distributions of plasma parameters: (a) $n_{e}$ and (b) $T_{e}$ for the different $V_{g}$ of $-10(\square),-20(\triangle)$, and $-30(\bigcirc) \mathrm{V}$ and for two different MF fields of $60(+)$ and $100(\nabla) \mathrm{G}$. Mesh size is $50 \mathrm{mesh} / \mathrm{in}$. The end plate is set at $z_{\text {end }}=-8 \mathrm{~cm}$. Experimental conditions: $V_{d}=50 \mathrm{~V}, I_{d}=2 \mathrm{~A}$, and $p\left(\mathrm{H}_{2}\right)=0.4 \mathrm{~Pa}$. 


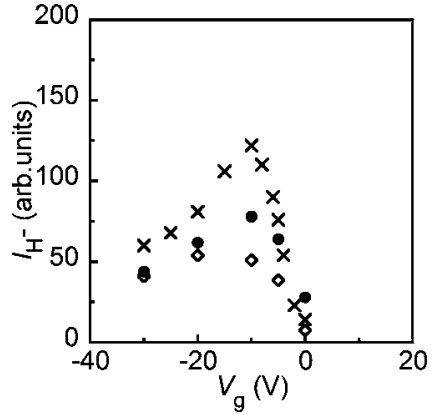

FIG. 4. Extracted negative ion currents as a function of $V_{g}$ for the different mesh sizes of No. $1(\diamond)$, No. $2(\bullet)$, and No. $3(\times)$. Experimental conditions: $V_{d}=50 \mathrm{~V}, I_{d}=2 \mathrm{~A}, p\left(\mathrm{H}_{2}\right)=0.4 \mathrm{~Pa}, z_{\text {end }}=-8 \mathrm{~cm}$, and extraction voltage $V_{\mathrm{ex}}=1 \mathrm{kV}$.

present case. Therefore, to increase the extracted negative ion currents, production of negative ions near the plasma end plate should be enhanced by optimizing plasma conditions.

According to the results shown in Fig. 3, the grid bias method is more suitable to optimize plasma conditions for negative ion production near the plasma grid, compared with the magnetic filter method.

\section{B. Negative ion production}

For negative ion volume production, it is expected that $T_{e}$ in the extraction region is maintained below $1 \mathrm{eV}$ with $n_{e}$ kept high. ${ }^{3}$ According to the results shown in Figs. 2 and 3, plasma parameters in the extraction region and then production of negative ions strongly depend on both $V_{g}$ and the position of the end plate $\left(z_{\text {end }}\right)$. Figure 4 shows the effect of $V_{g}$ on the $I_{\mathrm{H}^{-}}$(i.e., $\mathrm{H}^{-}$negative ion production). There is a certain optimum value of $V_{g}$ corresponding to the plasma conditions in the extraction region. Values of $I_{\mathrm{H}^{-}}$are different in mesh size [see Fig. 2(a)].

Figure 5 shows the effect of $z_{\text {end }}$ on the $I_{\mathrm{H}^{-}}$. At first, $I_{\mathrm{H}^{-}}$ increases by decreasing the distance between the MG and the end plate, reaches the maximum at $z_{\text {end }}=-4--5 \mathrm{~cm}$, and then decreases gradually. For the mesh bias method, there is a certain optimum position for negative ion extraction. On the other hand, for the magnetic filter method, the extracted negative ion current increases simply by decreasing the distance between the MF and the end plate. This difference is caused by the difference in plasma conditions in the extrac-

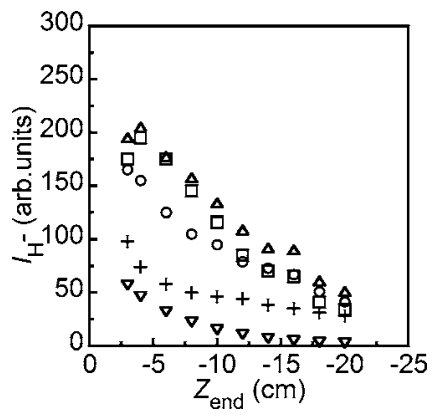

FIG. 5. Extracted negative ion currents as a function of the end plate position for two different cases, i.e., the mesh grid bias method with $V_{g}$ of -10 $(\square),-20(\triangle)$, and $-30(\bigcirc) \mathrm{V}$ and the magnetic filter method with the MF fields of $60(+)$ and $100(\nabla)$ G. Experimental conditions: $V_{d}=50 \mathrm{~V}$, $I_{d}=2 \mathrm{~A}, p\left(\mathrm{H}_{2}\right)=0.4 \mathrm{~Pa}$, and $V_{\mathrm{ex}}=1 \mathrm{kV}$.

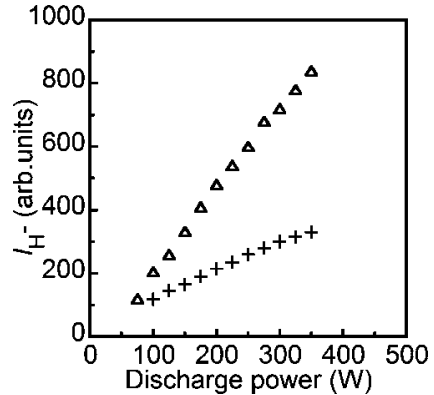

FIG. 6. Extracted negative ion currents as a function of discharge power for two different cases, i.e., the mesh grid bias method with $V_{g}$ of $-20(\triangle) \mathrm{V}$, and the magnetic filter method with the MF fields of $60(+) \mathrm{G}$. Experimental conditions: $V_{d}=50 \mathrm{~V}, p\left(\mathrm{H}_{2}\right)=0.33 \mathrm{~Pa}$, and $z_{\mathrm{end}}=-4 \mathrm{~cm}$ for the grid bias method. $V_{d}=50 \mathrm{~V}, p\left(\mathrm{H}_{2}\right)=0.27 \mathrm{~Pa}, z_{\text {end }}=-3 \mathrm{~cm}$ for the magnetic filter method, and $V_{\mathrm{ex}}=1 \mathrm{kV}$.

tion region. These characteristic behaviors are caused by the plasma parameters in the extraction region. Plasma production and then $\mathrm{H}^{-}$ion production also depend on hydrogen gas pressure $p\left(\mathrm{H}_{2}\right)$, although experimental results are not presented here.

Finally, some characteristic features of $\mathrm{H}^{-}$negative ion production is tested for two different methods of plasma parameter control, i.e., the grid bias method and the magnetic filter method. Figure 6 shows discharge power dependence of $I_{\mathrm{H}^{-}}$. According to the results shown in Fig. 5 and pressure dependence of the $\mathrm{H}^{-}$production, pressure and $z_{\text {end }}$ are optimized for two cases, respectively. With increasing power, $I_{\mathrm{H}^{-}}$ increases linearly and $I_{\mathrm{H}^{-}}$in the grid bias method is higher than the magnetic filter method.

The ion analyzer using the experiment consists of a $G_{1}$ electrode and a $G_{C}$ electrode. We observe that the extracted negative currents collected by the $G_{1}$ electrode is the extracted electron currents $\left(I_{e^{-}}\right)$and one collected by the $G_{C}$ electrode is the extracted negative ion currents $\left(I_{\mathrm{H}^{-}}\right)$, respectively. According to the results shown in Figs. 5 and $6, I_{\mathrm{H}^{-}}$ with the grid bias method is higher than that with magnetic filter method. However, $I_{e^{-}}$with the grid bias method is also higher than that with magnetic filter method (not shown here) because $n_{e}$ with the grid bias method is higher than that with magnetic filter method. In the future, we think that $I_{\mathrm{H}^{-}}$ is more extracted by suppressing the $I_{e^{-}}$with a certain transverse magnetic field.

\section{CONCLUSIONS}

Using the grid bias method, in dc discharge plasmas, control of plasma parameters and volume production of $\mathrm{H}^{-}$ negative ions are studied experimentally, and preliminary results are presented. We have confirmed that both high and low electron temperature plasmas are produced in the separated regions when the mesh grid is biased negatively. Plasma parameters in the extraction region and $\mathrm{H}^{-}$negative ion production depend on grid bias voltage. The extracted $\mathrm{H}^{-}$ current is higher than one with the MF method within the present experimental conditions. We hope that further study of controlling plasma parameter with the mesh bias method enhances negative ion production. As, in the future, rf nega- 
tive ion source is required for the NBI system, the grid bias method is quite useful to control and enhance negative ion volume production in rf plasmas.

\section{ACKNOWLEDGMENTS}

The authors would like to thank Professor Emeritus Noriyoshi Sato of Tohoku University, for his interest and encouragement to this work. They also thank Y. Tauchi for his assistance. A part of this work was supported by the Grant -in-Aid for Scientific Research (A) from Japan Society for the Promotion of Science.

${ }^{1}$ O. Fukumasa, J. Phys. D 22, 1668 (1989).

${ }^{2}$ O. Fukumasa, S. Mori, N. Nakada, Y. Tauchi, M. Hamabe, K. Tsumori, and Y. Takeiri, Contrib. Plasma Phys. 44, 516 (2004).
${ }^{3}$ O. Fukumasa and S. Mori, Nucl. Fusion 46, S287 (2006).

${ }^{4}$ O. Fukumasa and M. Matsumori, Rev. Sci. Instrum. 71, 935 (2000).

${ }^{5}$ T. Takanashi, Y. Takeiri, O. Kaneko, Y. Oka, K. Tsumori, and T. Kuroda, Rev. Sci. Instrum. 67, 1024 (1996).

${ }^{6}$ E. Speth, H. D. Falter, P. Franzen, U. Fantz, M. Bandyopadhyay, S. Christ, A. Enchera, M. Fröschle, D. Holtum, B. Hainemann, W. Kraus, A. Lorenz, Ch.. Martens, P. McNeely, S. Obermayer, R. Riedl, R. Süss, A. Tanga, R. Wilhelm, and D. Wünderlich, Nucl. Fusion 46, S220 (2006).

${ }^{7}$ K. Kato, S. Iizuka, and N. Sato, Appl. Phys. Lett. 65, 816 (1994).

${ }^{8}$ S. Iizuka, K. Kato, A. Takahashi, K. Nakagomi, and N. Sato, Jpn. J. Appl. Phys., Part 1 36, 4551 (1997).

${ }^{9}$ Y. Nakao, D. Ito, J. Ono, Y. Tauchi, and O. Fukumasa, Proceedings of the Sixth International Conference on Reactive Plasmas and 23rd Symposium on Plasma Processing, Matsushima/Sendai, 2006 (unpublished), p. 185.

${ }^{10}$ O. Fukumasa, D. Ito, and Y. Jyobira, 11th International Symposium on Production and Neutralization of Negative Ions and Beam, 2006 (unpublished).

${ }^{11}$ O. Fukumasa and R. Nishida, Nucl. Fusion 46, S220 (2006). 